\title{
X-ray Tomographic Microscopy of Drosophila Brain Network and Skeletonized Model Building in the Three-Dimensional Image
}

\author{
Ryuta Mizutani $^{1}$, Rino Saiga ${ }^{1}$, Kentaro Uesugi ${ }^{2}$, Akihisa Takeuchi $^{2}$, Yasuko Terada ${ }^{2}$ and Yoshio Suzuki ${ }^{2}$ \\ 1. Department of Applied Biochemistry, Tokai University, Hiratsuka, Kanagawa 259-1292, Japan. \\ 2. Japan Synchrotron Radiation Research Institute (JASRI/SPring-8), Sayo, Hyogo 679-5198, Japan.
}

The brain consists of a large number of neurons that make up a three-dimensional network. The first step to understanding brain functions is to analyze the structure of this network. Although three-dimensional structures of brain tissues have been reported, their structures are difficult to comprehend. This is because of a lack of quantitative descriptions of the three-dimensional network, which should be represented with three-dimensional Cartesian coordinates, rather than a three-dimensional distribution of intensities. Here, we report on x-ray tomographic microscopy of the brain network of the fruit fly Drosophila melanogaster and its analysis by skeletonized-model building [1].

Cephalic ganglion dissected from a wild-type Canton-S adult fly was stained with aurate by reducedsilver impregnation, as described previously [2,3]. The brain was then sequentially immersed in ethanol, $n$-butylglycidyl ether, and epoxy resin (Burnham Petrographics). The obtained sample was mounted using a nylon loop (Hampton Research) and incubated at $90^{\circ} \mathrm{C}$ for $16 \mathrm{~h}$ to cure the resin.

X-ray microtomography equipped with Fresnel zone plate (FZP) optics was performed at the BL37XU beamline of the SPring-8 synchrotron radiation facility. An FZP with an outermost zone width of 100 $\mathrm{nm}$ and diameter of $310 \mu \mathrm{m}$ was used as an x-ray objective lens. Transmission images produced by 8$\mathrm{keV}$ x-rays were recorded using a CMOS-based imaging detector (Hamamatsu Photonics). The tomographic slices were reconstructed from the x-ray images to determine the three-dimensional distribution of the linear attenuation coefficient. The spatial resolution was estimated to be $160 \mathrm{~nm}$ by using three-dimensional square-wave patterns. An example of the obtained structure is shown in Fig. 1. Neuronal processes were clearly visualized as a network structure.

In order to analyze the structure, its three-dimensional network should be further delineated in terms of Cartesian coordinates by building a skeletonized model. The model can be built by using a method like those used in crystallographic studies of macromolecular structures [1]. Since such an analysis should start by constructing an overall model that can facilitate structural analysis at a higher resolution, an initial model was built from a three-dimensional image obtained with other equipment of microtomography having a wider viewing field at BL47XU of SPring-8 [1]. Automatic tracing was applied to sparsely distributed structures such as those of peripheral nerves, while manual tracing was performed to build the neuropil model. The resulting model (Fig. 2) consists of neuronal processes with a total length of $378 \mathrm{~mm}$ in a volume of $0.220 \times 0.328 \times 0.314 \mathrm{~mm}^{3}$.

The neuronal process model was classified into groups on the basis of the three-dimensional structures. Anatomical segments can be extracted from the model by specifying neuronal processes in a group-bygroup manner. Figure 2 shows some of the structures of the optic lobe, which is responsible for visual information processing. Neuronal processes of the medulla and second optic chiasma, which are proximal to the compound eye, exhibit periodical structures corresponding to repeated units of photoreceptors. On the opposite side of the optic lobe, neuronal processes are assembled into several 
tracts pointed toward central brain regions. These structures represent information paths from the inputs on the eye to the integrative process in the central brain. Refinements of these models by using the high resolution data should reveal finer three-dimensional aspects of the Drosophila brain.

[1] R Mizutani et al, J. Struct. Biol. 184 (2013), 271-279.

[2] R Mizutani et al, Tissue Eng. C 14 (2008), 359-363.

[3] R Mizutani et al, J. Synchrotron Radiat. 15 (2008), 374-377.
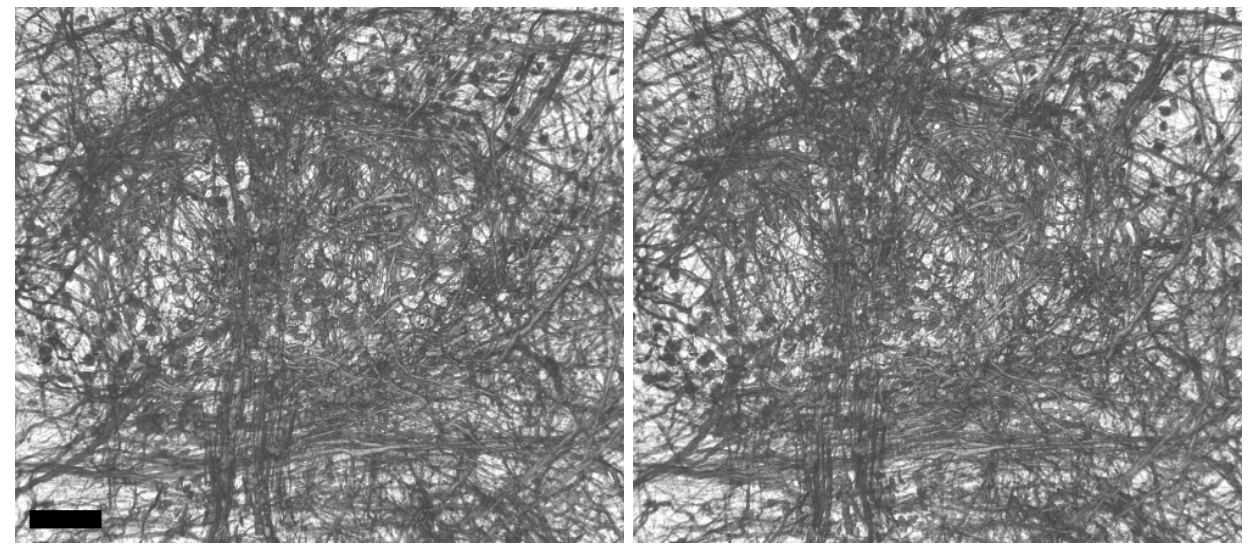

Figure 1. Stereo rendering of a frontal view of the Drosophila brain. Networks including the midline structure called the central complex are illustrated. Scale bar: $10 \mu \mathrm{m}$.

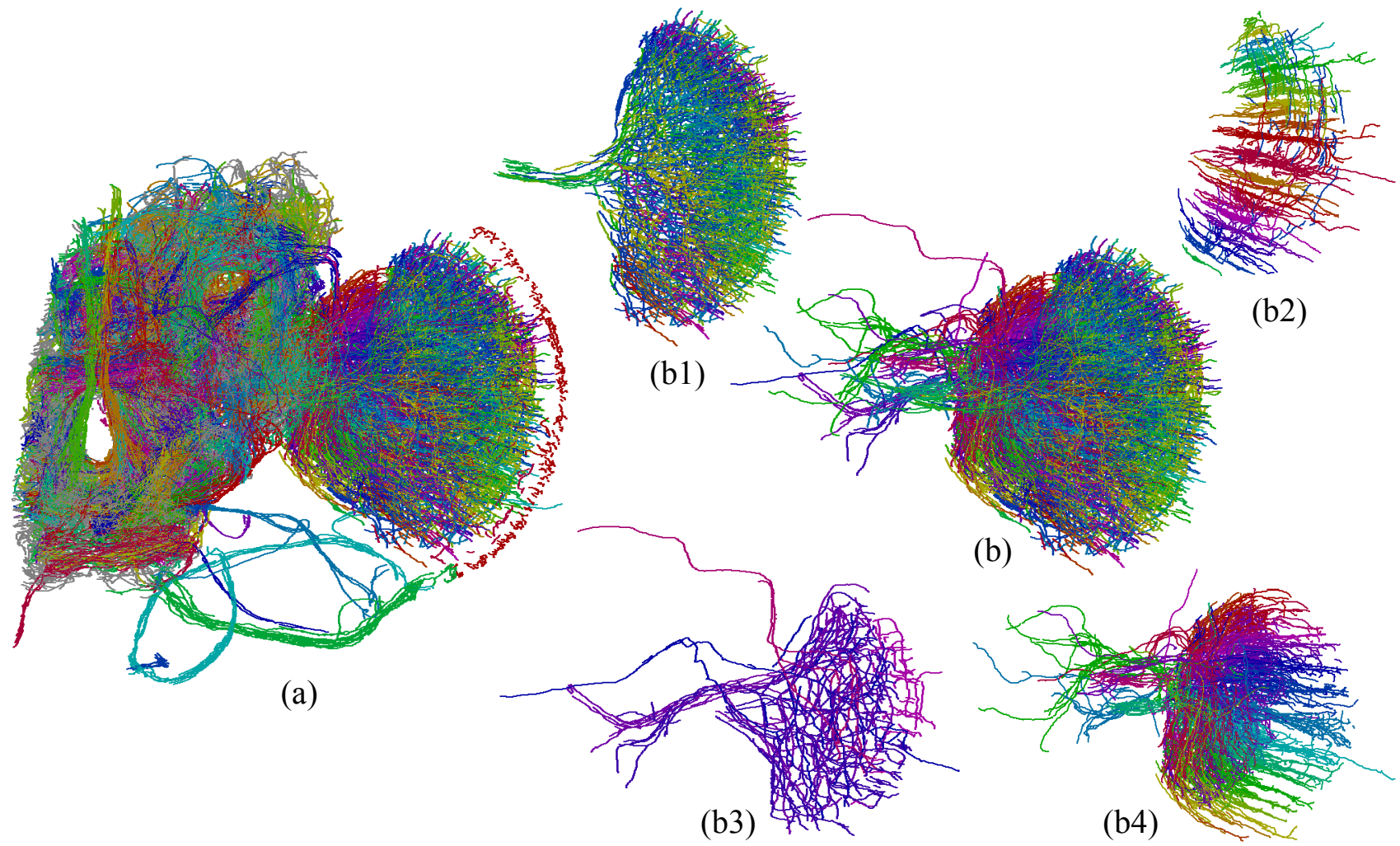

Figure 2. Skeletonized model of the left hemisphere of the Drosophila brain. The entire model is shown in (a). The optic lobe (b) is composed of the medulla (b1), second optic chiasma (b2), lobula plate (b3), and lobula (b4). Neuronal process groups are color-coded. 